\title{
How to perform high ultrasound examination of skin involvement among patients with systemic sclerosis - proposition of a unified protocol
}

\author{
Piotr Sobolewski ${ }^{1}{ }^{1 D}$, Monika Elżbieta Dźwigała ${ }^{1 D}$, Maria Maślińska ${ }^{2}{ }^{\text {ID }}$, Elżbieta Szymańska ${ }^{1}$ ID, \\ Irena Walecka ${ }^{1}$ ID \\ ${ }^{1}$ Clinic of Dermatology, Centre of Postgraduate Medical Education, Central Clinical Hospital of the Ministry of the Interior, \\ Warsaw, Poland \\ ${ }^{2}$ Clinic of Early Arthritis, National Institute of Geriatrics, Rheumatology and Rehabilitation, Warsaw, Poland
}

\begin{abstract}
Introduction: A fast and cheap method of skin assessment in systemic sclerosis (SSc) is an area of extensive research. Established in 1979, the Rodnan skin score is a palpation-based method used among clinicians. This method has some limitations, such as: examiner's skills, subjective results, and no standardization. In the last few years researchers have been exploring ultrasound-based techniques as a possible tool for skin assessment among patients with SSc. The aim of the study is to develop a protocol of elastography-based skin imaging evaluation among patients with SSc. Material and methods: Review of the literature and own experience.

Results: Proposition of elastography-based skin imaging protocol among patients with SSc.

Conclusions: The authors present a potential protocol of ultrasound-based examination of skin involvement among patients with SSc.
\end{abstract}

Key words: systemic sclerosis, ultrasound, protocol, Rodnan skin score.

\section{Introduction}

Systemic sclerosis (SSc) is a heterogeneous autoimmune disease, the etiology of which is still unknown [1]. There are two main types of SSc: limited (ISSc) and diffuse (dSSc) $[2,3]$. Skin involvement (mainly on hands, forearms and face) is characteristic for ISSc, whereas rapid skin and internal organ fibrosis is specific for dSSc [4].

The modified Rodnan skin score (mRSS) is a measure of skin thickness and is used in clinical settings and in clinical trials of SSc [5]. This is a palpation-based semi-quantitative score established in 1979 used for quantitative estimation of skin hardening [6]. Skin thickness is used as a marker of disease activity and mortality in patients with dSSc [6].

Moreover, increase of mRSS among patients with SSc is associated with negative renal and cardiac outcomes [6].
The modified Rodnan skin score is assessed by palpation of skin in 17 areas of the body (fingers, hands, forearms, arms, feet, legs and thighs, face, chest and abdomen) using a scale from 0 to 3, where 0 stands for normal, 1 for mild thickness, 2 for moderate thickness and 3 for severe thickness. Total skin score can range from 0 (normal skin without thickening) to 51 (hard skin with very severe thickening in all examined areas) [7].

Currently, ultrasound (US)-based techniques of skin imaging are being introduced as a potential tool to replace mRSS [8]. The following methods have been evaluated over the course of the last ten years and considered as tools for skin assessment in SSc: ultrasound elastography, shear-wave elastography (SWE), high-frequency ultrasonography (HFU), acoustic radiation force impulse (ARFI) imaging and magnetic resonance imaging (MRI) [9-12].

Address for correspondence:

Piotr Sobolewski, Clinic of Dermatology, Centre of Postgraduate Medical Education, Central Clinical Hospital of the Ministry of the Interior and Administration in Warsaw, 137 Wołoska St., 02-507 Warsaw, Poland, e-mail: piotr.sobolewski@cskmswia.pl, ORCID: https://orcid.org/0000-0002-3775-8021

Submitted: 06.01.2021; Accepted: 01.02.2021 


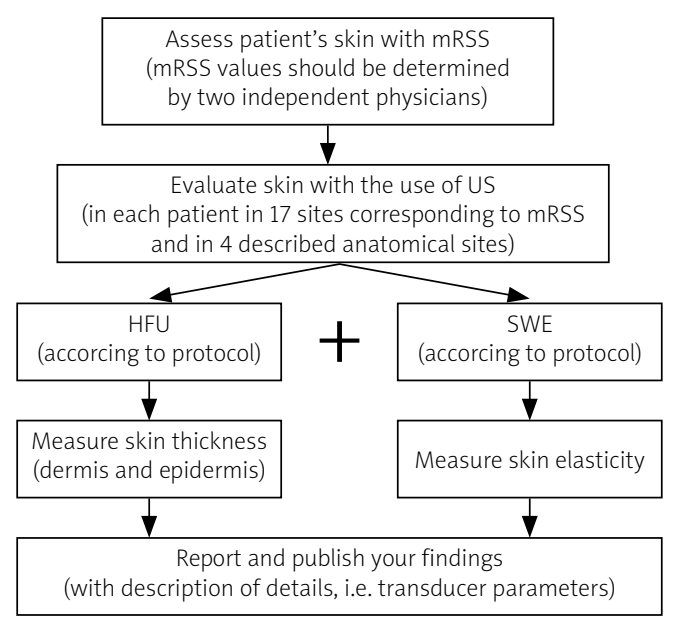

Fig. 1. Proposed protocol of skin examination in systemic sclerosis patients.

HFU - high-frequency ultrasonography, SWE - shear-wave elastography.

\section{Material and methods}

In the present article the authors propose a potential protocol to examine skin involvement among patients with SSc in order to obtain certain repeatability of results and findings (Figure 1). The authors suggest that in order to compare the results with each other researchers should apply the same protocol of examination; therefore, in this article the authors propose a unified protocol based on a literature review and personal experience.

Proposed protocol:

1. Assess patient's skin with mRSS, then evaluate skin by US applying HFU modality and SWE.

2. Measure skin thickness (including dermis and epidermis layers - describe each layer) when applying HFU and skin elasticity when applying SWE.

3. Perform two measurements using the US machine in each patient at 17 sites corresponding to mRSS and at 4 anatomical sites only (right and left middle fingers, right and left dorsal skin of forearms, the area between metacarpophalangeal joints II and III of the right hand, the dorsal skin of the proximal phalanx of the right and left second finger).

4. During the examination, cover skin at places of US examination with a $10-\mathrm{mm}$ hydrogel pad and a thick layer of gel. Place the transducer perpendicularly to the skin and obtain transverse images. Then, when the area of interest is clearly visualized, perform SWE (the elastography image should be stabilized). The region of interest (ROI) should be placed in the center of the screen and cover approximately $80 \%$ of the examined structure. Record three measurements for each $\mathrm{ROI}$ and record the average result.
5. If possible, verify the reproducibility of the results; RSS values should be determined by two independent physicians.

6. Report and publish your findings. Please record transducer parameters.

7. For patient comfort, carry out all examinations in a room with an ambient temperature of $20-25^{\circ} \mathrm{C}$, with the patient lying in a supine or prone position.

\section{Discussion}

Studies suggest that skin thickness (ST) measured with a US-based modality can replace mRSS examination [1, 9-12]. This is mainly because of the positive correlation between skin stiffness and the mRSS in these studies [1, 11, 13-19].

Skin involvement assessed with the mRSS correlates with skin stiffness and thickness. According to personal experience and analyzed studies the authors suggest the use of ultrasound-based techniques, in particular high-frequency ultrasound and shear-wave elastography $[9,10,13,14,17-20]$.

As many authors have already suggested, HFU allows one to measure dermal thickness and obtain comprehensive imaging of skin layers [9, 10, 13, 14, 17-20]. According to Sobolewski et al. [10] and Flower et al. [19] the HFU technique can distinguish skin layers through examination of ST, SWE and echogenicity. Some studies suggest that whole-body mRSS tended to correlate more closely to the finger or hand skin SE than ST among patients with SSc [20].

According to several authors, the mRSS correlated more positively with skin stiffness than with its thickness $[1,8,9,13,15,17]$; therefore, it is important to test both modalities among a larger group of patients. Interestingly, some authors reported a significant correlation between mRSS and US measurements in the phalanx area [14].

The results suggested that only one measurement at the above-mentioned location could potentially replace the mRSS. Such shortening of US-based examination to only one or two locations could possibly provide a cheap and easy method for clinicians. In order to reach an international consensus, the authors argue for the necessity of use of the same protocol among all physicians treating patients with SSC.

\section{Conclusions}

High-frequency ultrasonography can potentially surrogate the modified Rodnan skin score. There is a need for a unified examination protocol of ultrasound-based examinations. It is important to test which parameter is better to replace mRSS skin thickness or stiffness. 
The authors declare no conflict of interest.

\section{References}

1. Yang Y, Qiu L, Wang L, et al. Quantitative assessment of skin stiffness using ultrasound shear wave elastography in systemic sclerosis. Ultrasound Med Biol 2019; 45: 902-912, DOI: 10.1016/j.ultrasmedbio.2018.11.015.

2. Adigun R, Goyal A, Bansal P, Hariz A. Systemic sclerosis. In: StatPearls [Internet]. Treasure Island (FL): StatPearls Publishing 2020.

3. LeRoy EC, Black C, Fleischmajer R, et al. Scleroderma (systemic sclerosis): classification, subsets and pathogenesis. J Rheumatol 1988; 15: 202-205.

4. Johnson SR, van den Hoogen F, Devakandan K, et al. Systemic sclerosis: to subset or not to subset, that is the question. Eur J Rheumatol 2020; 7 (Suppl 3): S222-S227, DOI: 10.5152/ eurjrheum.2020.19116.

5. Ziemek J, Man A, Hinchcliff $M$, et al. The relationship between skin symptoms and the scleroderma modification of the health assessment questionnaire, the modified Rodnan skin score, and skin pathology in patients with systemic sclerosis. Rheumatology (Oxford) 2016; 55: 911-917, DOI: 10.1093/ rheumatology/kew003.

6. Khanna D, Furst DE, Clements PJ, et al. Standardization of the modified Rodnan skin score for use in clinical trials of systemic sclerosis. J Scleroderma Relat Disord 2017; 2: 11-18, DOI: 10.5301/jsrd.5000231.

7. Kaldas M, Khanna PP, Furst DE, et al. Sensitivity to change of the modified Rodnan skin score in diffuse systemic sclerosis-assessment of individual body sites in two large randomized controlled trials. Rheumatology (Oxford) 2009; 48 1143-1146, DOI: 10.1093/rheumatology/kep202.

8. Aryan A, Alaeen H, Dadgostar M, et al. Sonoelastography for skin evaluation in sclerodermic patients. Int J Prev Med 2019; 10: 91, DOI: 10.4103/ijpvm.IJPVM 43 18.

9. Li H, Furst DE, Jin $\mathrm{H}$, et al. High-frequency ultrasound of the skin in systemic sclerosis: an exploratory study to examine correlation with disease activity and to define the minimally detectable difference. Arthritis Res Ther 2018; 20: 181, DOI: 10.1186/s13075-018-1686-9.
10. Sobolewski P, Maślińska M, Zakrzewski J, et al. Applicability of shear wave elastography for the evaluation of skin strain in systemic sclerosis. Rheumatol Int 2020; 40: 737-745, DOI: 10.1007/s00296-020-04539-y.

11. Liu H, Hou Y, Zhu QL, et al. A preliminary study of skin ultrasound in diffuse cutaneous systemic sclerosis: does skin echogenicity matter? PLoS One 2017; 12: e0174481, DOI: 10.1371/ journal.pone. 0174481

12. Kang T, Abignano $G$, Lettieri $G$, et al. Skin imaging in systemic sclerosis. Eur J Rheumatol 2014; 1: 111-116, DOI: 10.5152/ eurjrheumatol.2014.036.

13. Yang Y, Yan F, Wang L, et al. Quantification of skin stiffness in patients with systemic sclerosis using real-time shear wave elastography: a preliminary study. Clin Exp Rheumatol 2018; 36 (Suppl 113): 118-125.

14. Sousa-Neves J, Cerqueira M, Santos-Faria D, et al. Ultrasound assessment of skin thickness performed on fingers: a tool for estimating overall severity of skin disease in systemic sclerosis patients? Acta Reumatol Port 2017; 42: 339-340.

15. Santiago T, Alcacer-Pitarch B, Salvador MJ, et al. A preliminary study using virtual touch imaging and quantification for the assessment of skin stiffness in systemic sclerosis. Clin Exp Rheumatol 2016; 34 (Suppl 100): 137-141.

16. Dinsdale G, Wilkinson S, Wilkinson J, et al. State-of-the-art technologies provide new insights linking skin and blood vessel abnormalities in SSc-related disorders. Microvasc Res 2020; 130: 104006, DOI: 10.1016/j.mvr.2020.104006.

17. Chen C, Cheng Y, Zhu X, et al. Ultrasound assessment of skin thickness and stiffness: the correlation with histology and clinical score in systemic sclerosis. Arthritis Res Ther 2020; 22: 197, DOI: 10.1186/s13075-020-02285-x.

18. Zhang X, Zhou B, Osborn T. Ultrasound surface wave elastography for assessing scleroderma. Ultrasound Med Biol 2020; 46: 1263-1269, DOI: 10.1016/j.ultrasmedbio.2020.01.021.

19. Flower VA, Barratt SL, Hart DJ, et al. High frequency ultrasound assessment of systemic sclerosis skin involvement: intraobserver repeatability and relationship with clinician assessment and dermal collagen content. J Rheumatol 2020, DOI: 10.3899/jrheum.200234 [Online ahead of print].

20. Naredo E, Pascau J, Damjanov N, et al. Performance of ultrahigh-frequency ultrasound in the evaluation of skin involvement in systemic sclerosis: a preliminary report. Rheumatology (Oxford) 2020; 59: 1671-1678, DOI: 10.1093/rheumatology/kez439. 